Proyecciones

Vol. 19, No 3, pp. 305-329, December 2000.

Universidad Católica del Norte

Antofagasta - Chile

DOI: $10.4067 /$ S0716-09172000000300006

\title{
EXISTENCE OF PERIODIC SOLUTIONS OF NEUTRAL FUNCTIONAL DIFFERENTIAL EQUATIONS WITH UNBOUNDED DELAY
}

\author{
HERNÁN R. HENRÍQUEZ * \\ Universidad de Santiago, Santiago-Chile.
}

\begin{abstract}
In this work we establish a result of existence of periodic solutions for quasi-linear partial neutral functional differential equations with unbounded delay on a phase space defined axiomatically.
\end{abstract}

Key words and phrases : Abstract Retarded Functional Differential Equations; Semigroups of linear operators.

*Partly supported by DICYT, Project 04-9133HM and by FONDECYT, Project 1970716. 


\section{Introduction}

In this note we establish a result of existence of periodic solutions for partial neutral functional differential equations with unbounded delay that can be modelled in the form

$$
\frac{d}{d t}\left(x(t)+F\left(t, x_{t}\right)\right)=A x(t)+G\left(t, x_{t}\right), \quad t \geq 0,
$$

where $A$ is the infinitesimal generator of a strongly continuous semigroup of linear operators on a Banach space $X$ and both $F$ as $G$ are appropriate functions. These equations will be called abstract neutral functional differential equations (abbreviated, ANFDE) with unbounded delay.

We refer the reader to [6] for a brief historical review and for the basic qualitative properties of the ANFDE with unbounded delay. Next, for completeness, we collect the notions that will be needed in section 2 .

Throughout this paper $X$ will be a Banach space provided with a norm $\|\cdot\|$ and $A: D(A) \subseteq X \rightarrow X$ will be the infinitesimal generator of a strongly continuous semigroup of linear operators $T(t)$ defined on $X$. For the theory of strongly continuous semigroups of linear operators we refer to Nagel [11] and Pazy [12]. We only recall here some notions and properties that will be essential for us. In particular, it is well known that there exist constants $\tilde{M} \geq 1$ and $\omega \in \mathbb{R}$ such that

$$
\|T(t)\| \leq \tilde{M} e^{\omega t}, \quad t \geq 0 .
$$

Moreover, if $T$ is an uniformly bounded and analytic semigroup with infinitesimal generator $A$ such that $0 \in \rho(A)$ (the resolvent set of $A$ ), then it is possible to define the fractional power $(-A)^{\alpha}$, for $0<\alpha \leq$ 1 , as a closed linear operator on its domain $D\left((-A)^{\alpha}\right)$. Furthermore, the subspace $D\left((-A)^{\alpha}\right)$ is dense in $X$ and the expression

$$
\|x\|_{\alpha}:=\left\|(-A)^{\alpha} x\right\|, \quad x \in D\left((-A)^{\alpha}\right)
$$

defines a norm on $D\left((-A)^{\alpha}\right)$. Hereafter we represent by $X_{\alpha}$ to the space $D\left((-A)^{\alpha}\right)$ endowed with the norm $\|\cdot\|_{\alpha}$. The following properties are well known $([12])$. 
Lemma 1.1 : Suppose that the preceding conditions are satisfied.

(a) Let $0<\alpha \leq 1$. Then $X_{\alpha}$ is a Banach space.

(b) If $0<\beta<\alpha \leq 1$ then $X_{\alpha} \hookrightarrow X_{\beta}$ and the imbedding is compact whenever the resolvent operator of $A$ is compact.

(c) For every $a>0$, there exists a positive constant $C_{a}$ such that

$$
\left\|(-A)^{\alpha} T(t)\right\| \leq \frac{C_{a}}{t^{\alpha}}, \quad 0<t \leq a .
$$

(d) For every $a>0$, there exists a positive constant $C_{a}^{\prime}$ such that

$$
\left\|(T(t)-I)(-A)^{-\alpha}\right\| \leq C_{a}^{\prime} t^{\alpha}, \quad 0<t \leq a
$$

To study equation (1.1) we assume that the histories $x_{t}:(-\infty, 0] \rightarrow$ $X, x_{t}(\theta):=x(t+\theta)$, belong to some abstract phase space $\mathcal{B}$, that is a phase space defined axiomatically.

In this work we will employ an axiomatic definition of the phase space $\mathcal{B}$ introduced by Hale and Kato [3]. To establish the axioms of space $\mathcal{B}$ we follow the terminology used in the book [8]. Thus, $\mathcal{B}$ will be a linear space of functions mapping $(-\infty, 0]$ into $X$ endowed with a seminorm $\|\cdot\|_{\mathcal{B}}$. We will assume that $\mathcal{B}$ satisfies the following axioms:

(A) If $x:(-\infty, \sigma+a) \rightarrow X, a>0$, is continuous on $[\sigma, \sigma+a)$ and $x_{\sigma} \in \mathcal{B}$ then for every $t$ in $[\sigma, \sigma+a)$ the following conditions hold:

(i) $x_{t}$ is in $\mathcal{B}$.

(ii) $\|x(t)\| \leq H\left\|x_{t}\right\|_{\mathcal{B}}$.

(iii) $\left\|x_{t}\right\|_{\mathcal{B}} \leq K(t-\sigma) \sup \{\|x(s)\|: \sigma \leq s \leq t\}+M(t-\sigma)\left\|x_{\sigma}\right\|_{\mathcal{B}}$.

Where $H \geq 0$ is a constant; $K, M:[0, \infty) \rightarrow[0, \infty), K$ is continuous and $M$ is locally bounded and $H, K$ and $M$ are independent of $x(\cdot)$.

(A-1) For the function $x(\cdot)$ in $(A), x_{t}$ is a $\mathcal{B}$-valued continuous function on $[\sigma, \sigma+a)$.

(B) The space $\mathcal{B}$ is complete.

We will denote by $\hat{\mathcal{B}}$ the quotient Banach space $\mathcal{B} /\|\cdot\|_{\mathcal{B}}$ and, if $\varphi \in \mathcal{B}$ we write $\hat{\varphi}$ for the coset determined by $\varphi$.

The axiom (A-1) implies that the operator functions $S(\cdot)$ and $W(t)$ 
given by

$$
[S(t) \varphi](\theta):=\left\{\begin{aligned}
\varphi(0), & -t \leq \theta \leq 0, \\
\varphi(t+\theta), & -\infty<\theta<-t,
\end{aligned}\right.
$$

and

$$
[W(t) \varphi](\theta):=\left\{\begin{aligned}
T(t+\theta) \varphi(0), & -t \leq \theta \leq 0, \\
\varphi(t+\theta), & -\infty<\theta<-t,
\end{aligned}\right.
$$

are strongly continuous semigroups of linear operators on $\mathcal{B}$.

Example 1.1 We consider the phase space $\mathcal{B}:=C_{r} \times L^{p}(g ; X), r \geq$ $0,1 \leq p<\infty$ in [8], which consists of all classes of functions $\varphi:(-\infty, 0] \rightarrow X$ such that $\varphi$ is continuous on $[-r, 0]$, Lebesguemeasurable and $g\|\varphi(\cdot)\|^{p}$ is Lebesgue integrable on $(-\infty,-r)$, where $g:(-\infty,-r) \rightarrow \mathbb{R}$ is a positive Borel measurable function (see Marle [9] for concepts regarded to the integration of vector valued functions). The seminorm in $\mathcal{B}$ is defined by

$$
\|\varphi\|:=\sup \{\|\varphi(\theta)\|:-r \leq \theta \leq 0\}+\left(\int_{-\infty}^{-r} g(\theta)\|\varphi(\theta)\|^{p} d \theta\right)^{1 / p} .
$$

Throughout this work we will assume that $g$ satisfies conditions (g-6) and $(g-7)$ in the terminology of [8]. This means that $g$ is integrable on $(-\infty,-r)$ and that there exists a nonnegative and locally bounded function $\gamma$ on $(-\infty, 0]$ such that

$$
g(\xi+\theta) \leq \gamma(\xi): g(\theta)
$$

for all $\xi \leq 0$ and $\theta \in(-\infty,-r) \backslash N_{\xi}$, where $N_{\xi} \subseteq(-\infty,-r)$ is a set with Lebesgue measure 0 . In this case, $\mathcal{B}$ is a phase space which verifies axioms (A), (A-1) and (B) ([8], Theorem 1.3.8).

In this note we will establish existence of solutions and existence of periodic solutions of equation (1.1), assuming that $T(\cdot), F$ and $G$ satisfy certain compactness conditions. Similar results, but based on the contraction mapping theorem have been established in [7].

Throughout this paper we always assume that $\mathcal{B}$ is a phase space. The terminology and notations are those generally used in operator 
theory. In particular, if $X$ and $Y$ denote Banach spaces, we indicate by $\mathcal{L}(X, Y)$ the Banach space of bounded linear operators from $X$ into $Y$ and we abbreviate this notation to $\mathcal{L}(X)$ whenever $X=Y$. In addition, we denote by $B_{r}[x]$ the closed ball with center at $x$ and radius $r$ and we reserve the bold type $\alpha$ to represent the Kuratowski's measure of non-compactness. For the properties of the measure $\alpha$ see Deimling [1].

\section{Existence of periodic solutions}

We begin by studying existence of mild solutions of the abstract Cauchy problem

$$
\begin{aligned}
\frac{d}{d t}\left(x(t)+F\left(t, x_{t}\right)\right) & =A x(t)+G\left(t, x_{t}\right), \quad t \geq \sigma, \\
x_{\sigma} & =\varphi \in \Omega,
\end{aligned}
$$

where $\Omega$ is an open subset of $\mathcal{B} ; \quad F, G:[\sigma, a] \times \Omega \rightarrow X$ are continuous functions and $0 \leq \sigma<a$.

Henceforth we will assume that $A$ is the infinitesimal generator of an analytic semigroup $T(\cdot)$ of bounded linear operators on $X$. In further, to avoid unnecessary notations, we suppose that $0 \in \rho(A)$ and that the semigroup $T(\cdot)$ is uniformly bounded, that is to say, $\|T(t)\| \leq \widetilde{M}$, for some constant $\widetilde{M} \geq 1$ and every $t \geq 0$.

Definition 2.1 : We will say that a function $x:(-\infty, \sigma+b) \rightarrow$ $X, b>0$, is a mild solution of the Cauchy problem (2.1)-(2.2) if $x_{\sigma}=\varphi$; the restriction of $x(\cdot)$ to the interval $[\sigma, \sigma+b)$ is continuous and for each $\sigma \leq t<\sigma+b$ the function $A T(t-s) F\left(s, x_{s}\right)$, $s \in[\sigma, t)$, is integrable and the integral equation

$x(t)=T(t-\sigma)[\varphi(0)+F(\sigma, \varphi)]-F\left(T, x_{t}\right)-\int_{\sigma}^{t} A T(t-s) F\left(s, x_{s}\right) d s$

$$
+\int_{\sigma}^{t} T(t-s) G\left(s, x_{s}\right) d s, \quad \sigma \leq t
$$


is verified.

Next we introduce some additional notations. For each pair of positive constants $\delta, r$ we define the set

$C(\sigma, \delta, r):=\{u \in C([\sigma, \sigma+\delta] ; X): u(\sigma)=0,\|u(t)\| \leq r, \sigma \leq t \leq$ $\sigma+\delta\}$.

It is easy to see that $C(\sigma, \delta, r)$ is a non-empty bounded, closed and convex subset of $C([\sigma, \sigma+\delta] ; X)$, whenever this space is provided with the sup norm. Furthermore, for $\varphi \in \mathcal{B}$ we will represent by $S(\varphi, \sigma, \delta, r)$ the set formed by the functions $x:(-\infty, \sigma+\delta] \rightarrow X$ such that $x_{\sigma}=\varphi, x_{t} \in \Omega, \sigma \leq t \leq \sigma+\delta, x(\cdot)$ is continuous on $[\sigma, \sigma+\delta]$ and $\sup _{\sigma \leq s \leq \sigma+\delta}\|x(s)-T(s-\sigma) \varphi(0)\| \leq r$. It is clear that if $\delta_{1} \leq \delta$ and $r_{1} \leq r$ then we may consider $S\left(\varphi, \sigma, \delta_{1}, r_{1}\right) \subseteq S(\varphi, \sigma, \delta, r)$. In the case $\sigma=0$ we shall abbreviate these notations by $C(\delta, r)$ and $S(\varphi, \delta, r)$, respectively. To relate these notations, we set $y(\cdot, \varphi):(-\infty, \infty) \rightarrow X$ for the function defined by

$$
y(t, \varphi):=\left\{\begin{aligned}
\varphi(t-\sigma), & -\infty<t<\sigma \\
T(t-\sigma) \varphi(0), & t \geq \sigma,
\end{aligned}\right.
$$

and $u(t)=x(t)-y(t, \varphi)$ for $x \in S(\varphi, \sigma, \delta, r)$ and $t \leq \sigma+\delta$. Since $u(t)=0$ for all $t \leq \sigma$ and $\|u(t)\| \leq r$ for $\sigma \leq t \leq \sigma+\delta$ we may consider $u \in C(\sigma, \delta, r)$. Moreover, in this case, $x_{t}=u_{t}+W(t-\sigma) \varphi$ for $\sigma \leq t \leq \sigma+\delta$. Conversely, for each $u \in C(\sigma, \delta, r)$ we denote by $\tilde{u}$ the extension of $u$ defined by $\tilde{u}(\theta)=0$, for $\theta \leq \sigma$, and $\tilde{u}(t):=u(t)$, for $\sigma \leq t \leq r$.

Initially we establish a result of existence of local solutions.

Theorem 2.1 : Let $\varphi \in \Omega$ and assume that the following conditions hold:

1. (a-1) There exists $\beta \in(0,1)$ such that the function $F$ is $X_{\beta^{-}}$ valued and $(-A)^{\beta} F$ is continuous .

(a-2) There are positive constants $\delta_{0}$ and $r_{0}$ such that the map $\widetilde{F}: C\left(\sigma, \delta_{0}, r_{0}\right) \rightarrow C\left(\left[\sigma, \sigma+\delta_{0}\right] ; X\right)$ given by $\widetilde{F}(u)(t):=F\left(t, \tilde{u}_{t}+\right.$ $W(t-\sigma) \varphi)$ is completely continuous .

(b-1) There exist constants $0<b(\varphi)<a-\sigma$ and $r(\varphi)>0$ such that $B_{r(\varphi)}[\varphi] \subseteq \Omega$ and, for each $0<t \leq b(\varphi)$, there is 
a compact set $U_{t} \subseteq X$ such that $T(t) G(s, \psi) \in U_{t}$, for all $\psi \in B_{r(\varphi)}[\varphi]$ and every $\sigma \leq s \leq \sigma+b(\varphi)$; then there exists a mild solution $x(\cdot, \varphi)$ of Cauchy problem (2.1)-(2.2) defined on $(-\infty, \sigma+b)$, for some $b>0$.

Proof. To simplify the notations we consider $\sigma=0$. In view of $(-A)^{\beta} F$ and $G$ are continuous functions and $\Omega$ is open in $\mathcal{B}$ we can assert that there exists $0<r^{\prime}<r(\varphi)$ such that $\left.B_{r^{\prime}}[\varphi] \subseteq \Omega ; \quad \|(-A)^{\beta} F(t, \psi)\right) \| \leq C_{1}$ and $\|G(t, \psi)\| \leq C_{2}$, for certain constants $C_{1}, C_{2} \geq 0$ and every $0 \leq t \leq b(\varphi)$ and $\psi \in B_{r^{\prime}}[\varphi]$. Since $W(\cdot) \varphi$ is continuous, we can choose $\delta^{\prime}>0$ such that

$$
\|W(t) \varphi-\varphi\|_{\mathcal{B}} \leq \frac{r^{\prime}}{2}
$$

for all $0 \leq t \leq \delta^{\prime}$. We set $\widetilde{K}:=\max _{0 \leq t \leq \delta^{\prime}} K(t)$ and $r:=$ $\min \left\{r_{0}, r^{\prime} /(2 \widetilde{K})\right\}$.

Since hypothesis (a-2) implies that the set of functions $\left\{F\left(t, x_{t}\right)\right.$ : $\left.x \in S\left(\varphi, \delta^{\prime}, r\right)\right\}$ is relatively compact then $\lim _{t \rightarrow 0} F\left(t, x_{t}\right)=F(0, \varphi)$, uniformly on $x \in S\left(\varphi, \delta^{\prime}, r\right)$. From this we derive that having fixed $\varepsilon>0$ small enough there exists $0<\delta \leq \min \left\{\delta^{\prime}, \delta_{0}, b(\varphi)\right\}$ for which the following hold:

and

$$
\begin{gathered}
\|(T(t)-I) F(0, \varphi)\| \leq \varepsilon, \\
\left\|F(0, \varphi)-F\left(t, x_{1}\right)\right\| \leq \varepsilon,
\end{gathered}
$$

for all $0 \leq t \leq \delta$, and $x(\cdot) \in S(\varphi, \delta, r)$, where $C_{a}$ denotes the constant introduced in Lemma 1.1(c).

If $x(\cdot)$ satisfies the equation (2.3), we can decompose it as $x(t)=u(t)+y(t, \varphi), t \leq \delta, \quad$ such as was indicated in the observations preceding the statement of this theorem. It is clear that the function $u(\cdot)$ verifies the equation

$$
\begin{gathered}
u(t)=T(t) F(0, \varphi)-F\left(t, u_{t}+y_{t}\right)- \\
\int_{0}^{t} A T(t-s) F\left(s, u_{s}+y_{s}\right) d s+\int_{0}^{t} T(t-s) G\left(s, u_{s}+y_{s}\right) d s \\
0 \leq t \leq \delta
\end{gathered}
$$


where we have abbreviated the notations by using $y(\cdot)$ instead of $y(\cdot, \varphi)$. This expression leads us to define the maps $\mathcal{T}, \mathcal{T}_{1}$, and $\mathcal{T}_{2}$ on $C(\delta, r)$ by means of

$$
\begin{gathered}
\left(\tau_{1} u\right)(t)=-\int_{0}^{t} A T(t-s) F\left(s, \widetilde{u}_{s}+y_{s}\right) d s, \\
\left(\tau_{2} u\right)(t)=T(t) F(0, \varphi)-F\left(t, \widetilde{u}_{t}+y_{t}\right)+ \\
\int_{0}^{t} T(t-s) G\left(s, \widetilde{u}_{s}+y_{s}\right) d s
\end{gathered}
$$

for $0 \leq t \leq \delta$ and $\mathcal{T}:=\mathcal{T}_{1}+\mathcal{T}_{2}$.

In what follows we will show that $\mathcal{T}_{1}$ and $\mathcal{T}_{2}$ are completely continuous maps with values in $C([0, \delta] ; X)$ and that $\mathcal{T}$ has compact range included in $C(\delta, r)$.

To prove these assertions, we observe initially that if $u(\cdot) \in$ $C(\delta, r)$ then $\tilde{u}_{t}+W(t) \varphi \in B_{r^{\prime}}[\varphi]$, for all $0 \leq t \leq \delta$. In fact, axiom (A) of the phase space and (2.4) yield that

$$
\begin{aligned}
\left\|\tilde{u}_{t}+W(t) \varphi-\varphi\right\|_{\mathcal{B}} & \leq\left\|\tilde{u}_{t}\right\|+\|W(t) \varphi-\varphi\|_{\mathcal{B}} \\
& \leq \widetilde{K} r+\frac{r^{\prime}}{2} \\
& \leq r^{\prime} .
\end{aligned}
$$

Now, since $G$ is a continuous function it is clear that $\mathcal{T}_{2}$ is well defined on $C(\delta, r)$ and that $\left(\mathcal{T}_{2} u\right)(\cdot)$ is also a continuous function. On the other hand, since $F$ is $X_{\beta}$-valued and $(-A)^{\beta} F$ is continuous then both $(-A)^{\beta} F\left(s, \tilde{u}_{s}+y_{s}\right)$ as $F\left(s, \tilde{u}_{s}+y_{s}\right)$ are continuous. In addition, in view of $T(\cdot)$ is an analytic semigroup ( see [12]), the operator function $s \rightarrow A T(t-s)$ is continuous in the uniform operator topology on $[0, t)$ and thus $A T(t-s) F\left(s, \tilde{u}_{s}+y_{s}\right)$ is also continuous on $[0, t)$. Applying the estimations established in Lemma 1.1 we obtain that

$$
\begin{gathered}
\left\|(-A) T(t-s) F\left(s, \tilde{u}_{s}+y_{s}\right)\right\|=\left\|(-A)^{1-\beta} T(t-s)(-A)^{\beta} F\left(s, \tilde{u}_{s}+y_{s}\right)\right\| \\
\leq \frac{C t e .}{(t-s)^{1-\beta}}
\end{gathered}
$$

which, by the Bochner's Theorem ([9]), implies that $\| A T(t-s) F\left(s, \tilde{u}_{s}+\right.$ $\left.y_{s}\right) \|$ is integrable on $[0, t)$. This concludes the proof that $\mathcal{T}_{1}$ is a well defined map with values in $C([0, \delta] ; X)$. 
Next we estimate $\|\mathcal{T} u(t)\|$ for $0 \leq t \leq \delta$. Since

$$
\begin{aligned}
\|(\mathcal{T} u)(t)\| \leq & \|(T(t)-I) F(0, \varphi)\|+\left\|F(0, \varphi)-F\left(t, \tilde{u}_{t}+y_{t}\right)\right\| \\
& +\left\|\int_{0}^{t}(-A)^{1-\beta} T(t-s)(-A)^{\beta} F\left(s, \tilde{u}_{s}+y_{s}\right) d s\right\| \\
& +\left\|\int_{0}^{t} T(t-s) G\left(s, \tilde{u}_{s}+y_{s}\right) d s\right\|
\end{aligned}
$$

and we can consider $u \in C\left(\delta_{0}, r\right)$, it is follows from (2.5), (2.6) and (2.7) that

$$
\begin{aligned}
\|(\mathcal{T} u)(t)\| & \leq 2 \varepsilon+\frac{C_{a} C_{1}}{\beta} \delta^{\beta}+\widetilde{M} C_{2} \delta \\
& \leq r
\end{aligned}
$$

which shows that $\mathcal{T}(u) \in C(\delta, r)$.

Next we will prove that the range of $\mathcal{T}_{1}$ is relatively compact. By Ascoli's theorem it is sufficient to show that the set $\mathcal{R}\left(\mathcal{T}_{1}\right)$ is equicontinuous on $[0, \delta]$ and $\mathcal{R}\left(\mathcal{T}_{1}\right)(t)$ is relatively compact in $X$ for each $0 \leq t \leq \delta$. We begin by showing this last assertion. Clearly, we may suppose that $t>0$. Let $0<\eta<t$. Then

$$
\begin{aligned}
\mathcal{T}_{1}(u)(t):= & -\int_{0}^{t-\eta} A T(\eta) T(t-\eta-s) F\left(s, \tilde{u}_{s}+y_{s}\right) d s \\
& +\int_{t-\eta}^{t}(-A)^{1-\beta} T(t-s)(-A)^{\beta} F\left(s, \tilde{u}_{s}+y_{s}\right) d s .
\end{aligned}
$$

From (a-2) we obtain that $F\left(s, \tilde{u}_{s}+y_{s}\right), 0 \leq s \leq \delta$, is included in a compact set and since $(-A) T(\eta)$ is a bounded operator, by the mean value theorem for the Bochner integral ([9]) we infer that the first term of the right hand side is also included in a compact set. Furthermore, since

$$
\left\|\int_{t-\eta}^{t}(-A)^{1-\beta} T(t-s)(-A)^{\beta} F\left(s, \tilde{u}_{s}+y_{s}\right) d s\right\| \leq \int_{t-\eta}^{t} \frac{C_{a} C_{1}}{(t-s)^{1-\beta}} d s
$$

converges to 0 , as $\eta \rightarrow 0$, we conclude that $\mathcal{R}\left(\mathcal{T}_{1}\right)(t)$ is relatively compact.

To prove the equicontinuity of $\mathcal{R}\left(\mathcal{T}_{1}\right)$ at $t_{0}$ we take $0 \leq t_{0}<t<\delta$. 
From the definition of $\mathcal{T}_{1}$ it follows that

$$
\begin{aligned}
\mathcal{T}_{1}(u)(t)-\mathcal{T}_{1}(u)\left(t_{0}\right)= & -\int_{0}^{t_{0}} A\left[T(t-s)-T\left(t_{0}-s\right)\right] F\left(s, \tilde{u}_{s}+y_{s}\right) d s \\
& -\int_{t_{0}}^{t} A T(t-s) F\left(s, \tilde{u}_{s}+y_{s}\right) d s \\
= & \left(T\left(t-t_{0}\right)-I\right) \mathcal{T}_{1}(u)\left(t_{0}\right) \\
& +\int_{t_{0}}^{t}(-A)^{1-\beta} T(t-s)(-A)^{\beta} F\left(s, \tilde{u}_{s}+y_{s}\right) d s .
\end{aligned}
$$

From this expression and using both the compactness of $\mathcal{R}\left(\mathcal{T}_{1}\right)\left(t_{0}\right)$ as the equi-integrability of functions $(-A)^{1-\beta} T(t-s)(-A)^{\beta} F\left(s, \tilde{u}_{s}+y_{s}\right)$, for $u \in C(\delta, r)$, we obtain that $\mathcal{R}\left(\mathcal{T}_{1}\right)$ is equicontinuous from the right at $t_{0}$. Proceeding similarly we can prove that $\mathcal{R}\left(\mathcal{T}_{1}\right)$ is equicontinuous at any $t_{0} \geq 0$.

On the other hand, using hypotheses (a-2) and (b-1), and repeating the argument (see also [4]) we can show that $\mathcal{R}\left(\mathcal{T}_{2}\right)$ is relatively compact in $C([0, \delta] ; X)$.

Finally, applying the Schauder's fixed point theorem we conclude the existence of a fixed point $u(\cdot) \in C(\delta, r)$ of $\mathcal{T}$. If we define $x(t)=\tilde{u}(t)+y(t),-\infty<t \leq \delta$, it is easy to see from the definition of $\mathcal{T}$ that $x(\cdot)$ is a mild solution of problem (2.1)-(2.2), which completes the proof of the theorem.

Proceeding as above we can also establish a result of existence of global solutions.

Corollary 2.1 : Suppose that $F$ and $G$ are defined on $[0, \infty) \times \mathcal{B}$ and that the hypotheses of Theorem 2.1 are verified for all $\sigma \geq 0$. Assume further that $F$ also satisfies the following condition :

(a-3) For every $\delta>0$ and all function $x:(-\infty, \delta) \rightarrow X$ such that $x_{0} \in \mathcal{B}$ and $x$ is continuous and bounded on $[0, \delta)$, the function $t \rightarrow$ $F\left(t, x_{t}\right)$ is uniformly continuous on $[0, \delta)$.

If $x(\cdot, \varphi):(-\infty, b), b>0$, is a noncontinuable solution of (2.12.2 ), with $\sigma=0$, which is bounded on $[0, b)$ then $b=\infty$. 
Proof. If we suppose that $b<\infty$, it follows from (a-3) that there exists $\lim _{t \rightarrow b^{-}} x(t, \varphi)$. Hence we obtain that the extension of $x(\cdot, \varphi)$ to $(-\infty, b]$ defined by $x(b):=\lim _{t \rightarrow b^{-}} x(t, \varphi)$ is continuous on $[0, b]$. Setting $\psi:=x_{b}$ the problem (2.1) with initial condition $\psi$ at $\sigma=b$ has a local solution $x(\cdot, \psi)$ defined on $(-\infty, b+\delta)$ for some $\delta>0$. It is easy to see from $(2.3)$ that $x(\cdot, \psi)$ is also a solution of the problem (2.1) with initial condition $x_{0}=\varphi$, which is contrary to our hypothesis.

In connection with these results it should be noted that if $(-A)^{\beta} F(t, \varphi)$ and $G(t, \varphi)$ satisfy further certain local Lipschitz or yet Hölder conditions on $\varphi$ then the mild solution of (2.1)-(2.2) is unique. Next, for completeness, we state a pair of results of this type. For the sake of brevity we omit their proofs.

Lemma 2.1 : Assume that for each $\varphi \in \Omega$ and each $\sigma \geq 0$ there exist positive constants $r, \delta, C_{1}$ and $C_{2}$ such that the following conditions hold:

(i) $\left\|(-A)^{\beta} F\left(t, \psi_{1}\right)-(-A)^{\beta} F\left(t, \psi_{2}\right)\right\| \leq C_{1}\left\|\psi_{1}-\psi_{2}\right\|_{\mathcal{B}}$;

(ii) $\left\|G\left(t, \psi_{1}\right)-G\left(t, \psi_{2}\right)\right\| \leq C_{2}\left\|\psi_{1}-\psi_{2}\right\|_{\mathcal{B}}$;

(iii) $C_{1} K(0)\left\|(-A)^{-\beta}\right\|<1$,

for $\sigma \leq t \leq \sigma+\delta$ and all $\psi_{1}, \psi_{2} \in B_{r}[\varphi]$. Then the mild solution of problem (2.1)-(2.2) is unique.

Lemma 2.2 : Assume that for each $\varphi \in \Omega$ and each $\sigma \geq 0$ there exist constants $C, \delta>0,0<\nu<1$ and continuous functions $k_{1}, k_{2}:[0, \infty) \rightarrow[0, \infty)$ such that the following conditions hold:

(i) $\left\|(-A)^{\beta} F\left(t, x_{t}^{1}\right)-(-A)^{\beta} F\left(t, x_{t}^{2}\right)\right\| \leq k_{1}(t-\sigma) \sup _{\sigma \leq s \leq t} \| x^{1}(s)-$ $x^{2}(s) \|^{\nu}$;

(ii) $\left\|G\left(t, x_{t}^{1}\right)-G\left(t, x_{t}^{2}\right)\right\| \leq k_{2}(t-\sigma) \sup _{\sigma \leq s \leq t}\left\|x^{1}(s)-x^{2}(s)\right\|^{\nu}$;

(iii) $k_{i}(t) \leq C t, i=1,2$,

for $\sigma \leq t \leq \sigma+\delta$ and all pair of functions $x^{1}, x^{2}:(-\infty, \sigma+\delta] \rightarrow X$ which are continuous on $[\sigma, \sigma+\delta]$ and $x_{0}^{1}=x_{0}^{2} \in \mathcal{B}$. Then the mild solution of problem (2.1)-(2.2) is unique.

In the sequel we assume that the functions $F, G$ and the semigroup $T(\cdot)$ satisfy appropriate conditions to assure existence and unique- 
ness of mild solutions of equation

$$
\frac{d}{d t}\left(x(t)+F\left(t, x_{t}\right)\right)=A x(t)+G\left(t, x_{t}\right), \quad t \geq 0,
$$

with initial condition

$$
x_{0}=\varphi \in \Omega .
$$

In these conditions we refer to (2.11) as an $\operatorname{ANFDE}(F, G)$ system. Furthermore, we will say that the $\operatorname{ANFDE}(\mathrm{F}, \mathrm{G})$ system is $\omega$-periodic if $F(t, \varphi)$ and $G(t, \varphi)$ are $\omega$-periodic at $t$. In the rest of this section we use $\omega$ to represent a fixed strictly positive constant.

Definition 2.2 : We will say that a function $x: \mathbb{R} \rightarrow X$ is an $\omega$-periodic solution of equation (2.11) if $x(\cdot)$ is a mild solution of (2.11) with initial condition $x_{0} \in \Omega$ and $x(t+\omega)=x(t)$, for all $t \geq 0$.

It is clear that if $x: \mathbb{R} \rightarrow X$ is a function such that $x_{0}=\varphi \in \mathcal{B}$, the restriction of $x(\cdot)$ on $[0, \omega)$ is continuous and $x_{\omega}=\varphi$ then $\varphi$ is $\omega$-periodic on $(-\infty, 0]$. In addition, if the $\operatorname{ANFDE}(\mathrm{F}, \mathrm{G})$ system is $\omega$-periodic and $x(\cdot, \varphi)$ is a mild solution of $(2.11)-(2.12)$ then the condition $x_{\omega}=\varphi$ is sufficient to guarantee that $x(\cdot, \varphi)$ is an $\omega$ periodic solution of (2.11). Since this is the essential property in order to obtain existence of periodic solutions we shall state it formally.

Proposition 2.1 : Assume that the $\operatorname{ANFDE}(\mathrm{F}, \mathrm{G})$ system is $\omega$ periodic and that the mild solution of (2.11) with initial condition $x_{0}=\varphi$ is defined on $\mathbb{R}$. If $x_{\omega}(\cdot, \varphi)=\varphi$ then $x(\cdot)$ is an $\omega$-periodic solution.

Let $E$ be a non empty closed subset of $\Omega$ such that the mild solution $x(\cdot, \varphi)$ of $(2.11)-(2.12)$ is unique and defined on $[0, \omega]$, for each $\varphi \in E$. In this case we represent by $P_{\omega}$ the map $E \rightarrow \mathcal{B}, \varphi \rightarrow$ $x_{\omega}(\cdot, \varphi)$.

If the $\operatorname{ANFDE}(\mathrm{F}, \mathrm{G})$ system is $\omega$-periodic, it is clear from the previous Proposition that a sufficient condition for the existence of a periodic solution of (2.11) is the existence of a fixed point for the map $P_{\omega}$. In order to establish the existence of a fixed point of $P_{\omega}$ we will apply the Sadovskii's Theorem. Nevertheless, an essential condition 
needed to apply this result is that the domain of the respective map it will be bounded, closed and convex. For this reason we introduce the following assumption.

Assumption (F,G): There exists a bounded, closed and convex set $E \subseteq \Omega$ such that for each $\varphi \in E$ the Cauchy problem (2.11)(2.12) has a unique mild solution $x(\cdot, \varphi)$ defined on $(-\infty, \omega]$, the closure of the set $\left\{x_{s}(\cdot, \psi): 0 \leq s \leq \omega, \psi \in E\right\}$ is bounded and included in $\Omega$ and $P_{\omega}(E) \subseteq E$.

Later we will exhibit a class of $\operatorname{ANFDE}(F, G)$ systems for which this assumption is valid. Since one of the hypotheses of the Sadovkii's theorem is the continuity of the respective map, our next result establish a criteria to assure the continuity of $P_{\omega}$.

Theorem 2.2 : Suppose that assumption $(F, G)$ holds. If we assume further that:

(a-4) There exists $\beta \in(0,1)$ such that $F$ is $X_{\beta}$-valued and the function $(-A)^{\beta} F$ is continuous and takes closed and bounded sets into bounded sets.

(a-5) For every $r>0$ and each $\varphi \in E$ the map $\widetilde{F}_{\varphi}: C(\omega, r) \rightarrow$ $C([0, \omega] ; X)$ given by $\tilde{F}_{\varphi}(u)(t):=F\left(t, \tilde{u}_{t}+W(t) \varphi\right)$ is completely continuous .

(b-2) The map $G$ takes closed and bounded sets into bounded sets and for each closed and bounded set $B \subseteq \Omega$ and each $t>0$ there exists a compact subset $\mathcal{W}_{t}$ of $X$ such that $T(t) G(s, \psi) \in \mathcal{W}_{t}$, for all $\psi \in B$ and every $0 \leq s \leq \omega$;

then the map $P_{\omega}: E \rightarrow \mathcal{B}, \varphi \rightarrow x_{\omega}(\cdot, \varphi)$ is continuous.

Proof. We begin by showing that for each relatively compact subset $B$ of $\mathcal{B}$ the set $\cup_{\varphi \in B} \mathcal{R}\left(\widetilde{F}_{\varphi}\right)$ is relatively compact in $C([0, \omega] ; X)$. In fact, from the continuity of $F$ and the compactness of the interval 
$[0, \omega]$ we obtain that for each $\varepsilon>0$ and each $\varphi \in \Omega$ there exists $\delta^{\prime}>0$ such that

$$
\sup _{0 \leq t \leq \omega}\|F(t, \varphi)-F(t, \psi)\| \leq \varepsilon,
$$

for all $\psi \in \Omega$ such that $0 \leq\|\varphi-\psi\|_{\mathcal{B}} \leq \delta^{\prime}$. Since for $u \in C(\omega, r)$, we have

$$
\begin{aligned}
\left\|\tilde{u}_{t}+W(t) \varphi-\tilde{u}_{t}-W(t) \psi\right\|_{\mathcal{B}} & =\|W(t)(\varphi-\psi)\|_{\mathcal{B}} \\
& \leq C_{1}\|\varphi-\psi\|_{\mathcal{B}}
\end{aligned}
$$

for all $0 \leq t \leq \omega$ and certain constant $C_{1}>0$, it follows that there is $\delta(\varphi)>0$ such that

$$
\sup _{0 \leq t \leq \omega}\left\|F\left(\tilde{u}_{t}+W(t) \varphi\right)-F\left(\tilde{u}_{t}+W(t) \psi\right)\right\|_{\mathcal{B}} \leq \varepsilon
$$

for $\|\varphi-\psi\|_{\mathcal{B}} \leq \delta(\varphi)$. Hence we can write

$$
\left\|\widetilde{F}_{\varphi}(u)-\widetilde{F}_{\psi}(u)\right\| \leq \varepsilon,
$$

for all $u \in C(\omega, r)$ and $\|\varphi-\psi\|_{\mathcal{B}} \leq \delta(\varphi)$. In view of the fact that $B$ is relatively compact, we can assert the existence of $\varphi^{1}, \varphi^{2}, \cdots, \varphi^{n}$ such that $B \subseteq \cup_{i=1}^{n} B_{\delta\left(\varphi^{i}\right)}\left[\varphi^{i}\right]$. From this we obtain that

$$
\begin{aligned}
\cup_{\varphi \in B} \mathcal{R}\left(\widetilde{F}_{\varphi}\right) & \subseteq \cup_{i=1}^{n} \cup_{u \in C(\omega, r)} B_{\varepsilon}\left[\widetilde{F}_{\varphi^{i}}\right] \\
& =\cup_{i=1}^{n} \mathcal{R}\left(\widetilde{F}_{\varphi^{i}}\right)+B_{\varepsilon}[0]
\end{aligned}
$$

Applying (a-5) we infer that the first set on the right hand side of the above inclusion is relatively compact . Since $\varepsilon$ was chosen arbitrarily this proves our asertion.

Let now $\left(\varphi^{n}\right)_{n}$ be a sequence in $E$ which converges to $\varphi$. We are going to prove that $P_{\omega} \varphi^{n}$ converges to $P_{\omega} \varphi$. Let $x^{n}:=x\left(\cdot, \varphi^{n}\right)$. First we will show that the set $\left\{x^{n}: n \in \mathbb{N}\right\}$ is relatively compact in $C([0, \omega] ; X)$. In fact, since $x^{n}(t)=z^{n}(t)-F\left(t, x_{t}^{n}\right)$, where

$$
\begin{aligned}
z^{n}(t):= & T(t)\left[\varphi^{n}(0)+F\left(0, \varphi^{n}\right)\right]-\int_{0}^{t} A T(t-s) F\left(s, x_{s}^{n}\right) d s \\
& +\int_{0}^{t} T(t-s) G\left(s, x_{s}^{n}\right) d s, \quad 0 \leq t \leq \omega,
\end{aligned}
$$

and by the Assumption (F, G) the set $\left\{x_{t}^{n}: 0 \leq t \leq \omega, n \in \mathbb{N}\right\}$ is bounded, we may proceed as in the proof of Theorem 2.1 to show that 
$\left\{z^{n}(\cdot): n \in \mathbb{N}\right\}$ is relatively compact in $C([0, \omega] ; X)$. Furthermore, turning to use the boundedness of the set $\left\{x_{t}^{n}: 0 \leq t \leq \omega, n \in \mathbb{N}\right\}$ we obtain that there is $r>0$ for which

$$
\left\{F\left(t, x_{t}^{n}\right): n \in \mathbb{N}\right\} \subseteq \cup_{n \in N} \mathcal{R}\left(\widetilde{F}_{\varphi^{n}}\right)
$$

which implies the set $\left\{F\left(t, x_{t}^{n}\right): n \in \mathbb{N}\right\}$ is relatively compact in $C([0, \omega] ; X)$. This completes the proof of this asertion.

Thus we can find a subsequence of $x^{n}(\cdot)$, denoted with the same index, which converges to $u \in C([0, \omega] ; X)$. We define the function $\tilde{u}$ on $(-\infty, \omega]$ by $\tilde{u}(\theta):=\varphi(\theta)$, for $\theta \leq 0$, and $\tilde{u}(\theta):=u(t)$, for $0 \leq t \leq \omega$. It is follows from the axioms of phase space that $x_{t}^{n} \rightarrow \tilde{u}_{t}$, as $n \rightarrow \infty$, and the Lebesgue's dominated convergence theorem implies that

$$
\begin{aligned}
u(t)= & T(t)[\varphi(0)+F(0, \varphi)]-F\left(t, \tilde{u}_{t}\right)-\int_{0}^{t} A T(t-s) F\left(s, \tilde{u}_{s}\right) d s \\
& +\int_{0}^{t} T(t-s) G\left(s, \tilde{u}_{s}\right) d s, \quad 0 \leq t \leq \omega .
\end{aligned}
$$

Therefore, $\tilde{u}(\cdot)$ is the mild solution of (2.11)-(2.12) and $P_{\omega}(\varphi)=\tilde{u}_{\omega}=$ $\lim _{n \rightarrow \infty} x_{\omega}^{n}=\lim _{n \rightarrow \infty} P_{\omega}\left(\varphi^{n}\right)$. In view of the fact that this conclusion remains valid whenever we replace $\left(\varphi^{n}\right)_{n}$ by any of its subsequences we infer that $P_{\omega}$ is continuous .

We are now in conditions to establish the main result of this section.

Theorem 2.3 : Assume that (a-4) and (a-5) are satisfied. If further the following conditions are fulfilled:

(a) The $\operatorname{ANFDE}(F, G)$ system is $\omega$-periodic and the assumption (F, G) holds.

(b) The semigroup $T(\cdot)$ is compact.

(c) The function $G:[0, \infty) \times \Omega \rightarrow X$ takes bounded and closed sets into bounded sets.

(d) There exists $\sigma \in(0, \omega)$ such that

$$
M(\omega-\sigma)\left[K(\sigma) \sup _{0 \leq s \leq \sigma}\|T(s)\| H+M(\sigma)\right]<1 .
$$

Then the equation (2.11) has an $\omega$-periodic solution. 
Proof. Since $T(\cdot)$ is a compact semigroup and $G(\cdot)$ takes bounded and closed sets into bounded sets, it follows from Theorem 2.2 that the map $P_{\omega}$ ( in short, $P$ ) : $E \rightarrow E, \quad \varphi \rightarrow x_{\omega}(\cdot, \varphi)$ is continuous . Consequently, there exists an induced map $\hat{P}: \hat{E} \rightarrow \hat{E}$ which satisfies the condition $\hat{P}(\hat{\varphi})=\widehat{P(\varphi)}$, for every $\hat{\varphi} \in \hat{E}$ and every $\varphi \in \hat{\varphi}$. Our objective is to show that $\hat{P}$ is a condensing map. Since for each subset $C$ of $\hat{E}$ there is $D \subseteq E$ such that $C=\hat{D}$ and $\boldsymbol{\alpha}(C)=\boldsymbol{\alpha}(D)$, we restrict our attention to estimate the value $\boldsymbol{\alpha}(\hat{P}(\hat{D}))$, for each $D \subseteq E$ with $\boldsymbol{\alpha}(D)>0$.

It will be convenient for our purpose to introduce some notations. Let $0 \leq \sigma_{1} \leq \sigma_{2} \leq \omega$. We define the following sets of functions defined on $\left[\sigma_{1}, \sigma_{2}\right]$ :

$$
\begin{aligned}
D\left[\sigma_{1}, \sigma_{2}\right] & :=\left\{\left.x(\cdot, \varphi)\right|_{\left[\sigma_{1}, \sigma_{2}\right]}: \varphi \in D\right\} \\
D_{1}\left[\sigma_{1}, \sigma_{2}\right] & :=\left\{\left.T(\cdot) \varphi(0)\right|_{\left[\sigma_{1}, \sigma_{2}\right]}: \varphi \in D\right\} \\
D_{2}\left[\sigma_{1}, \sigma_{2}\right] & :=\left\{\left.z(\cdot, \varphi)\right|_{\left[\sigma_{1}, \sigma_{2}\right]}: z(t, \varphi)=x(t, \varphi)-T(t) \varphi(0), \varphi \in D\right\} .
\end{aligned}
$$

It is clear that $D\left[\sigma_{1}, \sigma_{2}\right]$ and $D_{i}\left[\sigma_{1}, \sigma_{2}\right], i=1,2$, are subsets of $C\left(\left[\sigma_{1}, \sigma_{2}\right] ; X\right)$. Furthermore,

$$
D\left[\sigma_{1}, \sigma_{2}\right] \subseteq D_{1}\left[\sigma_{1}, \sigma_{2}\right]+D_{2}\left[\sigma_{1}, \sigma_{2}\right]
$$

On the other hand, for $0 \leq \sigma \leq \omega$ we set $D_{\sigma}:=\left\{x_{\sigma}(\cdot, \varphi): \varphi \in D\right\}$.

Proceeding as in the proof of Theorem 2.2 we obtain that $D_{2}\left[\sigma_{1}, \sigma_{2}\right]$ is a relatively compact subset of $C\left(\left[\sigma_{1}, \sigma_{2}\right] ; X\right)$ so that

$$
\boldsymbol{\alpha}\left(D\left[\sigma_{1}, \sigma_{2}\right]\right) \leq \boldsymbol{\alpha}\left(D_{1}\left[\sigma_{1}, \sigma_{2}\right]\right) .
$$

Furthermore, since the semigroup $T$ is compact, $\boldsymbol{\alpha}\left(D_{1}\left[\sigma_{1}, \sigma_{2}\right]\right)=0$, for $\sigma_{1}>0$ and

$$
\boldsymbol{\alpha}\left(D_{1}[0, \sigma]\right) \leq \sup _{0 \leq s \leq \sigma}\|T(s)\| H \boldsymbol{\alpha}(D)
$$

On the other hand, combining the previous estimations with the The- 
orem 2.1 in [14] we obtain that

$$
\begin{aligned}
\boldsymbol{\alpha}(\hat{P}(\hat{D})) & \leq K(\omega-\sigma) \boldsymbol{\alpha}(D[\sigma, \omega])+M(\omega-\sigma) \boldsymbol{\alpha}\left(\hat{D}_{\sigma}\right) \\
& \leq M(\omega-\sigma)[K(\sigma) \boldsymbol{\alpha}(D[0, \sigma])+M(\sigma) \boldsymbol{\alpha}(D)] \\
& \leq M(\omega-\sigma)\left[K(\sigma) H \sup _{0 \leq s \leq \sigma}\|T(s)\|+M(\sigma)\right] \boldsymbol{\alpha}(D),
\end{aligned}
$$

for each $0<\sigma \leq \omega$ which, by condition (d), implies that $\hat{P}$ is a condensing map. Finally, by the Sadovskii's fixed point theorem ([13]), we infer that $P$ has a fixed point in $E$ and, based in our previous discussion, we can assert that there exists an $\omega$-periodic solution of (2.11).

In practical applications the property $K(\cdot)$ bounded and $M(t)$ convergent towards zero when $t$ goes to infinity is frequently encountered (see [8]). For this reason, next we present a pair of consequences of Theorem 2.3 in the context of phase spaces verifying such property. In this case, and without any danger of confussion, we will employ the same symbol $K$ to denote $\sup _{t \geq 0} K(t)$. First we present a class of systems which satisfy the Assumption $(F, G)$.

Proposition 2.2 : Assume that the functions $F, G:[0, \infty) \times$ $\mathcal{B} \rightarrow X$ satisfy conditions (a-3), (a-4), (a-5) and (b-2). Suppose, furthermore, that the following conditions hold:

(i) The function $K(\cdot)$ is bounded and $M(t) \rightarrow 0$ as $t \rightarrow \infty$;

(ii) There is $\mu>0$ such that $\|T(t)\| \leq \widetilde{M} e^{-\mu t}$, for $t \geq 0$;

(iii) There exist positive constants $N_{1}, N_{2}, N_{3}$ and $N_{4}$ such that

$$
\left\|(-A)^{\beta} F(t, \varphi)\right\| \leq N_{1}\|\varphi\|_{\mathcal{B}}+N_{2}
$$

and

$$
\|G(t, \varphi)\| \leq N_{3}\|\varphi\|_{\mathcal{B}}+N_{4}
$$

then for $N_{1}$ and $\frac{N_{3}}{\mu}$ small enough and for every $\varphi \in \mathcal{B}$ the mild solution $x(\cdot, \varphi)$ is defined and bounded on $\mathbb{R}$ and the Assumption (F, $\mathrm{G})$ is fulfilled.

Proof. Let $x=x(\cdot, \varphi)$ be the mild solution of (2.1-2.2) corresponding to $\sigma=0$. From Theorem 2.1 we obtain that $x$ is defined 
on $(-\infty, b)$, for some $b>0$. Applying (2.3) we infer the existence of positive constants $C_{1}$ and $C_{2}$ which are independent of $\varphi$ such that

$$
\left\|x_{t}\right\|_{\mathcal{B}} \leq K\left(C_{1} e^{-\mu t}\|\varphi\|_{\mathcal{B}}+C_{2}\right)+M(t)\|\varphi\|_{\mathcal{B}}
$$

for $0 \leq t<b$. This estimation and Theorem 2.2 show that $x$ is defined and bounded on $\mathbb{R}$. Furthermore, it is clear from this estimation that if we choose $R>C_{2} K$ and $\omega$ large enough then $\left\|x_{\omega}\right\|_{\mathcal{B}} \leq R$ for all $\varphi \in \mathcal{B}$ such that $\|\varphi\|_{\mathcal{B}} \leq R$. Thus, the Assumption $(\mathrm{F}, \mathrm{G})$ is verified with $E=B_{R}[0]$.

Corollary 2.2 : Assume that $F, G$ and $T$ satisfy the conditions $(\mathrm{a}-3),(\mathrm{a}-4)$ and $(\mathrm{a}-5)$ and conditions (a), (b), (c) considered in of Theorem 2.3. If further $K(\cdot)$ is a bounded function and $M(t) \rightarrow 0$, as $t \rightarrow \infty$, then (2.11) has an $m \omega$-periodic solution for some $m \in \mathbb{N}$.

Proof. We apply Theorem 2.3 on an interval $[0, m \omega]$. It is clear that for $m$ large enough, by taking $\sigma:=m \omega / 2$, the condition (d) of the already mentioned result is fulfilled.

We complete this work applying our results to the study of existence of periodic solutions of a concrete equation. Initially, we present some general examples.

Example 2.1 : If the function $F$ satisfies the following condition: (a-6) There are bounded functions $L_{1}, L_{2}:[0, \omega] \rightarrow[0, \infty)$ with $L_{1}(h), \quad L_{2}(h) \rightarrow 0, \quad$ as $h \rightarrow 0$, and a locally bounded function $f:$ $[0, \infty) \rightarrow[0, \infty)$ such that

$$
\begin{array}{r}
\left\|F\left(t, x_{t}\right)-F\left(s, x_{s}\right)\right\| \leq L_{1}(t-s) f\left(\left\|x_{s}\right\|_{\mathcal{B}}\right)+L_{2}(t-s) \sup \{f(\|x(\xi)\|) \\
: s \leq \xi \leq t\},
\end{array}
$$

for $0 \leq s \leq t<b$ and for every function $x:(-\infty, b) \rightarrow X$ which is continuous on $[0, b)$ and $x_{t} \in \Omega$, for $0 \leq t<b$, then $F$ satisfies condition (a-3). If, in further, $F(t, \cdot)$ is completely continuous for each $0 \leq t \leq \omega$, then $F$ also verifies (a-5).

Working in concrete phase spaces it is not difficult to present examples of $\operatorname{ANFDE}(F, G)$ systems which verify the conditions considered in the previous results. 
Next we study a linear operator in the space $\mathcal{B}:=C_{r} \times L^{p}(g ; X)$, with $r=0$, defined in the Example 1.1.

Example 2.2. Let $\mathcal{B}=C_{r} \times L^{p}(g ; X)$, with $r=0$ and $p>1$, be the space defined in Example 1.1. We set

$$
\Lambda(t, \varphi):=\int_{-\infty}^{0} C(t, \theta) \varphi(\theta) d \theta,
$$

where $C(t, \theta) \in \mathcal{L}(X)$ is a strongly measurable map defined on $[0, \infty) \times$ $(-\infty, 0]$ which satisfies the following conditions:

(i) For each $t, \theta, C(t, \theta)$ is a compact linear operator and this property is verified locally uniformly at $\theta$. This means that for every $s>0$ the set $\{C(t, \theta) x:\|x\| \leq 1,-s \leq \theta \leq 0\}$ is relatively compact in $X$;

(ii) For each $t \geq 0$, the function $\frac{\|C(t, \theta)\|}{g(\theta)^{1 / p}}$ is $q$-integrable on $(-\infty, 0]$ and

(ii-1) $\sup _{0 \leq t \leq b} \int_{-\infty}^{0} \frac{\|C(t, \theta)\|^{q}}{g(\theta)^{q-1}} d \theta<\infty ;$

$$
L_{1}(h):=\sup _{0 \leq t \leq \omega}\left(\int_{-\infty}^{0}\left\|\frac{C(t+h, \theta-h)}{g(\theta)^{1 / p}}-\frac{C(t, \theta)}{g(\theta)^{1 / p}}\right\|^{q} d \theta\right)^{1 / q} \rightarrow 0, h \rightarrow 0^{+},
$$

for every $b>0$. where $q$ denotes the conjugate exponent of $p$.

Then $\Lambda$ satisfies conditions (a-3) and (a-5). In fact, condition (ii-1) implies that $\Lambda$ is well defined and $\Lambda(t, \cdot)$ is a bounded linear map from $\mathcal{B}$ into $X$. Moreover, $\Lambda(t, \cdot)$ is a compact operator . In fact, setting, for each $s>0$,

$$
\Lambda_{s}(t, \varphi)=\int_{-s}^{0} C(t, \theta) \varphi(\theta) d \theta
$$

the same argument already used shows that $\Lambda_{s}(t, \cdot)$ is a bounded linear operator from $\mathcal{B}$ into $X$ and Theorem 1 in [5] implies that $\Lambda_{s}(t, \cdot)$ is compact . Since $\Lambda_{s}(t, \cdot)$ converges uniformly to $\Lambda(t, \cdot)$ as $s \rightarrow \infty$ it follows that $\Lambda(t, \cdot)$ is compact .

On the other hand, if $x(\cdot) \in S(\varphi, b, r)$, then it is clear that

$$
\begin{aligned}
\left\|\Lambda\left(t, x_{t}\right)-\Lambda\left(s, x_{s}\right)\right\| \leq & \int_{-\infty}^{0}\|C(s+h, \theta-h)-C(s, \theta)\|\|x(s+\theta)\| d \theta \\
& +\int_{-h}^{0}\|C(s+h, \theta)\|\|x(s+h+\theta)\| d \theta \\
\leq & L_{1}(h)\left\|x_{s}\right\|_{\mathcal{B}}+L_{2}(h) \sup _{s \leq \xi \leq t}\|x(\xi)\|
\end{aligned}
$$


where we have denoted

$$
L_{2}(h):=\sup _{0 \leq t \leq b}\left(\int_{-h}^{0}\left\|\frac{C(t, \theta)}{g(\theta)^{1 / p}}\right\|^{q} d \theta\right)^{1 / q}\left(\int_{-h}^{0} g(\theta) d \theta\right)^{1 / p}
$$

and $h:=t-s$ Thus, (ii-2) and the integrability of $g$ show that condition (a-6) is verified so that the assertion is consequence of previous example.

Related with this example and Proposition 2.2 it is worth to point out that if $g$ satisfies (g-6) and (g-7) in the terminolgy of [8] and $\gamma(-t) \rightarrow 0$, as $t \rightarrow \infty$, then $K(\cdot)$ is a bounded function and $M(t) \rightarrow 0$ as $t \rightarrow \infty$ ([8], Theorem 1.3.7 and Example 7.1.8). In the example that follows we suppose $g$ verifies these conditions as well as that $\ln g$ is uniformly continuous .

Example 2.3 We conclude this section with an application of our results to discuss the existence of periodic solutions of the boundary value problem

$$
\begin{array}{r}
\frac{\partial}{\partial t}\left[u(t, \xi)+\int_{-\infty}^{t} \int_{0}^{\pi} b(s-t, \eta, \xi) q(u(s, \eta)) d \eta d s\right]=\frac{\partial^{2}}{\partial \xi^{2}} u(t, \xi)+ \\
a_{0}(\xi) u(t, \xi)+\int_{-\infty}^{t} a(s-t) u(s, \xi) d s+a_{1}(t, \xi), \\
t \geq 0,0 \leq \xi \leq \pi,
\end{array}
$$

where the functions $a_{0}, a, a_{1}, b, q$ and $\phi$ satisfy appropriate conditions. To represent this problem as the Cauchy problem (2.1)-(2.2) we shall take $X:=L^{2}([0, \pi])$ and define $x(t):=u(t, \cdot)$. The operator $A$ is given by

$$
A f(\xi):=f^{\prime \prime}(\xi)
$$

with domain

$$
D(A):=\left\{f(\cdot) \in L^{2}([0, \pi]): f^{\prime \prime}(\cdot) \in L^{2}([0, \pi]), \quad f(0)=f(\pi)=0\right\} .
$$


It is well known that $A$ generates a strongly continuous semigroup $T(\cdot)$ which is compact, analytic, self-adjoint and uniformly stable. Specifically, $\|T(t)\| \leq e^{-t}, t \geq 0$. Furthermore, $A$ has discrete spectrum, the eigenvalues are $-n^{2}, n \in \mathbb{N}$, with corresponding normalized eigenvectors $z_{n}(\xi):=\left(\frac{2}{\pi}\right)^{1 / 2} \sin (n \xi)$. The operator $(-A)^{1 / 2}$ is defined by

$$
(-A)^{1 / 2} f=\sum_{n=1}^{\infty} n<f, z_{n}>z_{n}
$$

on the space $D\left((-A)^{1 / 2}\right)=\left\{f(\cdot) \in X: \sum_{n=1}^{\infty} n<f, z_{n}>z_{n} \in X\right\}$.

Let $\mathcal{B}$ denote the space $C_{r} \times L^{2}(g ; X)$, with $r=0$, defined in Example 1.1. In this case (see [8]) $H=1 ; K(t)=1+\left(\int_{-t}^{0} g(\theta) d \theta\right)^{1 / 2}$ and $M(t)=\gamma(-t)^{1 / 2}$ for all $t \geq 0$. It is clear that $\mathcal{B}$ is isomorphic and isometric to the space $X \times L_{\mu}^{2}((-\infty, 0] \times[0, \pi])$ where $\mu$ is the measure $\mu(\theta, \xi)=g(\theta) d \theta d \xi$. Next we assume that the following conditions hold:

(i) The function $b(\cdot)$ is measurable and

(i-1) For each $r>0$, $\sup _{-r \leq \theta \leq 0} \int_{0}^{\pi} \int_{0}^{\pi} b^{2}(\theta, \eta, \xi) d \eta d \xi<\infty$;

(i-2) For each $r>0, \lim _{h \rightarrow 0} \int_{0}^{\pi} \int_{0}^{\pi} \mid b(\theta, \eta, \xi+h)-$

$\left.b(\theta, \eta, \xi)\right|^{2} d \eta d \xi=0$ uniformly on $\theta \in[-r, 0]$;

(i-3) $\int_{0}^{\pi} \int_{-\infty}^{0} \int_{0}^{\pi} \frac{b^{2}(\theta, \eta, \xi)}{g(\theta)} d \eta d \theta d \xi<\infty$.

(ii) The function $\frac{\partial}{\partial \zeta} b(\theta, \eta, \zeta)$ is measurable; $b(\theta, \eta, \pi)=0$; $b(\theta, \eta, 0)=0$ and

$$
N:=\int_{0}^{\pi} \int_{-\infty}^{0} \int_{0}^{\pi} \frac{1}{g(\theta)}\left(\frac{\partial}{\partial \zeta} b(\theta, \eta, \zeta)\right)^{2} d \eta d \theta d \zeta<\infty
$$

(iii) The function $a_{0}(\cdot) \in L^{\infty}([0, \pi]) ; a(\cdot)$ is measurable with $\int_{-\infty}^{0} \frac{a^{2}(\theta)}{g(\theta)} d \theta<\infty$ and $a_{1}(t, \cdot) \in L^{2}([0, \pi])$ for each $t \geq 0$ and the function $t \rightarrow a_{1}(t, \cdot)$ is continuous.

(iv) The function $q: \mathbb{R} \rightarrow \mathbb{R}$ is Lipschitz or Hölder continuous and $|q(\xi)| \leq C_{1}|\xi|+C_{2}$ for some constants $C_{1}, C_{2} \geq 0$.

(v) The function $\varphi$ defined by $\varphi(\theta)(\xi):=\phi(\theta, \xi)$ belongs to $\mathcal{B}$.

Under these conditions we define $F, G:[0, \infty) \times \mathcal{B} \rightarrow X$ by $F(t, \varphi):=\Lambda_{1} \circ Q(\varphi)$ and $G(t, \varphi):=\Lambda_{2}(\varphi)+h(t)$, where 


$$
\begin{gathered}
Q(\varphi)(\theta, \xi):=q(\varphi(\theta, \xi)), \\
\Lambda_{1}(\varphi)(\xi)=\int_{-\infty}^{0} \int_{0}^{\pi} b(\theta, \eta, \xi) \varphi(\theta, \eta) d \eta d \theta, \\
\Lambda_{2}(\varphi)(\xi):=a_{0}(\xi) \varphi(0, \xi)+\int_{-\infty}^{0} a(\theta) \varphi(\theta, \xi) d \theta, \\
h(t)=a_{1}(t, \cdot),
\end{gathered}
$$

for $0 \leq \xi \leq \pi$. A straithforward estimation using (i) and (iii) shows that $\Lambda_{1}$ and $\Lambda_{2}$ are bounded linear operators on $\mathcal{B}$ and that the function $h(\cdot)$ is continuous . Furthermore, the values $\Lambda_{1}(\varphi) \in D\left((-A)^{1 / 2}\right)$ and $\left\|(-A)^{1 / 2} \Lambda_{1}\right\| \leq N^{1 / 2}$. In fact, from (2.20) and (ii) it follows that

$$
<\Lambda_{1}(\varphi), z_{n}>=\frac{1}{n}\left(\frac{2}{\pi}\right)^{1 / 2}<\Lambda(\varphi), \cos (n \zeta)>
$$

where $\Lambda$ is defined by

$$
\Lambda(\varphi):=\int_{-\infty}^{0} \int_{0}^{\pi} \frac{\partial}{\partial \zeta} b(\theta, \eta, \zeta) \varphi(\theta, \eta) d \eta d \theta
$$

From (ii) we obtain that $\Lambda: \mathcal{B} \rightarrow X$ is a bounded linear operator with $\|\Lambda\| \leq N^{1 / 2}$. Hence we can write $\left\|(-A)^{1 / 2} \Lambda_{1}(\varphi)\right\|=\|\Lambda(\varphi)\|$ which implies the assertion.

Therefore, the system (2.16-2.17-2.18) satisfies conditions (a-4) and (b-2). Now we will show that $F$ verifies the hypotheses of Example 2.2. To this end we define $C(\theta)$ on $L^{2}([0, \pi])$ by

$$
[C(\theta) f](\xi) ;=\int_{0}^{\pi} b(\theta, \eta, \xi) f(\eta) d \eta
$$

It is clear that

$$
\Lambda_{1}(\varphi)=\int_{-\infty}^{0} C(\theta) \varphi(\theta) d \theta
$$

From (i-1) and Exercise VI.9.52 in [2] we conclude that $C(\theta)$ is a compact linear operator from $L^{2}([0, \pi])$ into $L^{2}([0, \pi])$ with

$$
\|C(\theta)\| \leq\left(\int_{0}^{\pi} \int_{0}^{\pi} b^{2}(\theta, \eta, \xi) d \xi d \eta\right)^{1 / 2} .
$$


Moreover, from (i-1), (i-2) and the characterization of relatively compact sets in $L^{2}([0, \pi])([2]$, Theorem IV.8.20) it follows that the operators $C(\theta)$ are locally uniformly compacts . The condition (i-3) implies that $\frac{\|C(\theta)\|}{g(\theta)^{1 / 2}}$ is 2 -integrable on $(-\infty, 0]$. Finally we can estimate $L_{1}(h)$ as follows

$$
\begin{aligned}
L_{1}(h) & =\int_{-\infty}^{0} \frac{\|C(\theta-h)-C(\theta)\|^{2}}{g(\theta)} d \theta \\
& =\int_{-\infty}^{0}\left\|\frac{g_{1}(\theta-h)}{g_{1}(\theta)} \frac{C(\theta-h)}{g_{1}(\theta-h)}-\frac{C(\theta)}{g_{1}(\theta)}\right\|^{2} d \theta \\
& \leq \quad 2 \int_{-\infty}^{0} \frac{g(\theta-h)}{g(\theta)}\left\|\frac{C(\theta-h)}{g_{1}(\theta-h)}-\frac{C(\theta)}{g_{1}(\theta)}\right\|^{2} d \theta+2 \int_{-\infty}^{0} \mid \frac{g_{1}(\theta-h)}{g_{1}(\theta)}- \\
& \left.1\right|^{2} \frac{\|C(\theta)\|^{2}}{g(\theta)} d \theta,
\end{aligned}
$$

where we have abbreviated the notation setting $g_{1}=g^{1 / 2}$. Using the properties of $g$ and the integrability of $\frac{\|C(\theta)\|^{2}}{g(\theta)}$ we obtain that the right hand side of the above inequality converges to zero as $h \rightarrow 0$. From Example 2.1 and 2.2 we derive that $\Lambda_{1}$ verifies conditions (a-3) and (a-5). On the other hand, $Q$ is a substitution operator which is continuous and takes bounded sets into bounded sets ([10]). Moreover, the Lipschits or Hölder continuity of $q$ implies that $Q$ has the same property, respectively. Since $F$ is a Hammerstein operator formed by the composition of the linear operator $\Lambda_{1}$ and the operator $Q$ we obtain that $F$ satisfies the properties already established for $\Lambda_{1}$. Thus, the system (2.16-2.17-2.18) verifies conditions (a-3) and (a-5). In addition, it is not difficult to see that $F$ satisfies the hypotheses considered in Lemma 2.1 or Lemma 2.2 so that for each $\phi$ there is a unique mild solution $x(\cdot, \phi)$ defined on $(-\infty, b)$, for some $b>0$. Furthermore, if $C_{1}\left\|\Lambda_{1}\right\|$ and $\left\|\Lambda_{2}\right\|$ are small enough and $h$ is bounded then the solution $x(\cdot, \phi)$ is $\|\cdot\|_{2}$-bounded on $[0, \infty)$.

In connection with the existence of periodic solutions, if $h$ is $\omega$ periodic, from Proposition 2.2 and Corollary 2.2 we obtain that for $C_{1}\left\|\Lambda_{1}\right\|$ and $\left\|\Lambda_{2}\right\|$ enough small there exists an $m \omega$-periodic mild solution. 


\section{References}

[1] K. Deimling, Non Linear Functional Analysis, Springer-Verlag, Berlin, (1985).

[2] N. Dunford and J. T. Schwartz, Linear Operators. Part I, John Wiley and Sons, New York, (1988).

[3] J. Hale and J. Kato, Phase space for retarded equations with infinite delay, Funkcialaj Ekvac., 21, pp. 11-41, (1978).

[4] H. R. Henríquez, Periodic solutions of quasi-linear partial functional differential equations with unbounded delay. Funkcialaj Ekvac. 37 (2), pp. 329-343, (1994).

[5] H. R. Henríquez, On non-exact controllable systems, Int. J. Control, 42(1), pp. 71-83, (1985).

[6] E. Hernández and H. R. Henríquez, Existence Results for partial neutral functional differential equations with unbounded delay, J. Math. Anal. Appl. 221, pp. 452-475, (1998).

[7] E. Hernández and H. R. Henríquez, Existence of periodic solutions of partial neutral functional differential equations with unbounded delay. J. Math. Anal. Appl., 221, pp. 499-522, (1998).

[8] Y. Hino, S. Murakami and T. Naito, Functional Differential Equations with Infinite Delay, Lect. Notes in Maths., 1473. SpringerVerlag, Berlin, (1991).

[9] C-M. Marle, Mesures et Probabilités, Hermann, Paris, (1974).

[10] R. H. Martin, Nonlinear Operators $\&$ Differential Equations in Banach Spaces, Robert E. Krieger Publ. Co., Florida, (1987).

[11] R. Nagel, One-parameter Semigroups of Positive Operators, Lect. Notes in Maths. 1184 (editor), Springer-Verlag, Berlin, (1986).

[12] A. Pazy, Semigroups of Linear Operators and Applications to Partial Differential Equations, Springer-Verlag, New York, (1983). 
[13] B. N. Sadovskii, On a fixed point principle. Funct. Anal. Appl., 1, pp. 74-76, (1967).

[14] S. J. Shin, An existence theorem of a functional differential equation, Funkcialaj Ekvac. 30, pp. 19-29, (1987).

Received : October 2000.

\section{Hernán R. Henríquez Miranda}

Departamento de Matemática

Universidad de Santiago de Chile

Casilla 307

Correo 2

Santiago

Chile

e-mail : henrique@fermat.usach.cl 